\title{
Chaetomugilin J Enhances Apoptosis in Human Ovarian Cancer A2780 Cells Induced by Cisplatin Through Inhibiting PinkI/Parkin Mediated Mitophagy
}

This article was published in the following Dove Press journal: OncoTargets and Therapy

\author{
Xiaoqing $\mathrm{Hu} \mathbb{D}^{1,2, *}$ \\ Jiabin Wang $\mathbb{D}^{1, *}$ \\ Jiannan Chai ${ }^{3}$ \\ Xiaoya $\mathrm{Yu}^{4}$ \\ Yunhan Zhang' \\ Yuqi Feng (D) \\ Jianchun $Q i^{5}$ \\ Huimei $\mathrm{Yu}^{\prime}$ \\ 'Key Laboratory of Pathobiology, \\ Ministry of Education, Department of \\ Pathophysiology, College of Basic Medical \\ Sciences, Jilin University, Changchun, Jilin \\ I3002 I, People's Republic of China; \\ ${ }^{2}$ Department of Ophthalmology, First \\ Hospital of Jilin University, Changchun, \\ Jilin I3002 I, People's Republic of China; \\ ${ }^{3}$ Department of Clinical Laboratory, First \\ Hospital of Jilin University, Changchun, \\ jilin I3002I, People's Republic of China; \\ ${ }^{4}$ Jilin Provincial Science and Technology \\ Innovation Platform Management Center, \\ Changchun, Jilin 1300 I2, People's \\ Republic of China; ${ }^{5}$ College of Plant \\ Sciences, Jilin University, Changchun, Jilin \\ I30062, People's Republic of China
}

*These authors contributed equally to this work

Correspondence: Jianchun Qin College of Plant Sciences, Jilin University, Changchun, Jilin I30062, People's Republic of China

Email qinjc@jlu.edu.cn

Correspondence: Huimei Yu Key Laboratory of Pathobiology, Ministry of Education, Department of Pathophysiology, College of Basic Medical Sciences, Jilin University, Changchun, Jilin I3002I, People's Republic of China

Email yuhuimei@jlu.edu.cn
Purpose: The chemoresistance and toxicity of traditional chemotherapeutic drugs have become obstacles to their antitumor effects in ovarian cancers. Therefore, it is particularly important to develop new anticancer drugs to increase target sensitivity and reduce the toxicity of chemotherapy drugs. As key organelles, the endoplasmic reticulum and mitochondria play important role in chemoresistance. Cells become resistant to drugs by maintaining the homeostasis of the endoplasmic reticulum and mitochondria. Chaetomugilin J, a metabolite isolated from Polygonatum sibiricum, belongs to the Chaetomium family and exhibits potent cytotoxicity. In this study, we aimed to explore the mechanistic link between apoptosis and endoplasmic reticulum stress, mitophagy and mitochondrial dysfunction induced by chaetomugilin $\mathrm{J}$ combined with cisplatin in the ovarian cancer cell line A2780.

Methods: Chaetomugilin J was identified by chemical methods. Cell viability was measured by an MTT assay. The apoptosis, mitochondrial membrane potential, and intracellular reactive oxygen species (ROS) were examined by flow cytometry. Mitochondrial ROS was measured by a fluorescence microscope with MitoSox staining. Further, the related proteins and overexpression of parkin were detected by Western blot.

Results: Chaetomugilin J combined with low-dose cisplatin decreased cell viability and increased apoptosis in A2780 cells. In addition, intracellular ROS and mitochondrial ROS were increased, while the mitochondrial membrane potential was reduced. The expressions of grp78 and chop were decreased after treatment by chaetomugilin J combined with lowdose cisplatin. Overexpression of parkin attenuated chaetomugilin J combined with cisplatininduced apoptosis.

Conclusion: Chaetomugilin $\mathrm{J}$ combined with cisplatin inhibited pink1/parkin mediated mitophagy increased mitochondrial dysfunction in the A2780 cells and enhanced apoptosis induced by cisplatin in the ovarian cancer cell line A2780. But this process was not related to endoplasmic reticulum apoptotic pathway.

Keywords: chaetomugilin J, cisplatin, mitophagy, apoptosis, ovarian cancer

\section{Introduction}

Ovarian cancer is one of the most common gynecological malignancies, among which epithelial ovarian cancer (EOC) is the most lethal. ${ }^{1}$ Although most patients are sensitive to platinum chemotherapy drugs at the beginning of treatment, treatment extension could lead to chemoresistance, which in turn, can lead to disease recurrence. ${ }^{2,3}$ Therefore, it is urgent to explore new drugs that can increase the sensitivity of ovarian cancer cells to cisplatin. It has been found that many natural 
compounds derived from plants have antitumor activity, which have advantages such as low genotoxicity., Chaetomugilin is an endophytic Chaetomium globosum strain from Polygonatum sibiricum. These compounds have been reported to exhibit cytotoxic activity in 39 human cancer cell lines. ${ }^{6,7}$ However, whether chaetomugilin can increase the sensitivity of ovarian cancer cells to cisplatin needs to be studied further.

The avoidance of apoptosis is an important reason for cisplatin resistance in ovarian cancer. ${ }^{8,9}$ Apoptosis is associated with endoplasmic reticulum stress or mitochondrial damage. ${ }^{10}$ The endoplasmic reticulum is the primary organelle responsible for a variety of cellular functions, including protein folding, maturation, and the maintenance of homeostasis. ${ }^{11}$ Endoplasmic reticulum stress triggers unfolded protein response (UPR), which is one of the pathways leading toward apoptosis. ${ }^{12}$ It has been reported that cisplatin promotes cell apoptosis through endoplasmic reticulum stress. ${ }^{13,14}$ Mitochondria are organelles that are closely related to apoptosis and chemotherapy resistance, and have become the targets of many antitumor drugs. ${ }^{15}$ In the mitochondrial apoptotic pathway, mitochondria release apoptotic factors such as cytochrome c, which activate the caspase cascade reaction. These caspases then cleave other proteins such as PARP-1 and induce apoptosis. ${ }^{16}$

Mitophagy is the selective removal of damaged or unnecessary mitochondria using autophagic machinery, ${ }^{17}$ and plays an essential part in maintaining mitochondrial homeostasis. Mitophagy, as a kind of autophagy, may play different roles in various environments and stress conditions. ${ }^{18,19}$ On the one hand, mitophagy is involved in the quality control of mitochondria and can protect cells by clearing damaged mitochondria. On the other hand, it can induce autophagic cell death or promote apoptosis by regulating many pathways. For example, Chen et al reported that activating pink1/parkinmediated mitophagy by downregulating COX-2, consequently resulting in apoptosis and thereby inhibiting the growth of HCC. ${ }^{20}$ Therefore, exploring the effect of mitophagy on apoptosis might help us identify a new target drug to treat diseases.

In the present study, we observed that chaetomugilin J, either alone or in combination with cisplatin, exhibits antitumor activity in the ovarian cancer cell line A2780. In particular, chaetomugilin $\mathrm{J}$ significantly reduced the concentration of cisplatin when inducing apoptosis in A2780 cells. Cell apoptosis was not related to the endoplasmic reticulum apoptotic pathway. Our results showed that chaetomugilin J, combined with cisplatin, mainly targeted the mitochondria, impaired their normal function, and inhibited mitophagy in terms of activating the mitochondrial apoptotic pathway.

\section{Materials and Methods Culture and Isolation}

The strain of C. globosum was initially isolated from the root of $P$. sibiricum. We collected plants and stored at $4^{\circ} \mathrm{C}$ for no more than 2 weeks. Then we selected fresh roots with intact plant samples and washed them with sterile water to remove the soil on the surface. They were purified under sterile conditions. The fungal strain was cultured at $28^{\circ} \mathrm{C}$ for 40 days in a liquid medium $(50 \mathrm{~L})$ containing soluble starch $1 \%$ and casein $0.1 \%$ in $50 \%$ artificial seawater.

\section{Preparation of Chaetomugilin J}

The culture was filtered under suction and the mycelia collected were extracted thrice with $\mathrm{MeOH}$. The combined extracts were evaporated in vacuo to give a mixture of crude metabolites (25.6 g), the $\mathrm{CHCl}_{3}-\mathrm{MeOH}$ (1:1) soluble fraction of which exhibited cytotoxicity. The culture filtrate was extracted thrice with AcOEt. The combined extracts were evaporated in vacuo to afford a mixture of crude metabolites $(19.8 \mathrm{~g})$ that exhibited cytotoxicity. The AcOEt extract was passed through silica gel column using $\mathrm{CHCl}_{2}-\mathrm{MeOH}$ as the eluent. The second fraction $(7.2 \mathrm{~g})$ in which the activity was concentrated was chromatographed on a Sephadex $\mathrm{LH}-20$ with a $\mathrm{CHCl}_{3}-\mathrm{MeOH}(1: 1)$ as the eluent. The $\mathrm{CHCl}_{3}$ eluate $(55.4 \mathrm{mg}$ ) was purified by HPLC using $\mathrm{MeON}-\mathrm{H}_{2} \mathrm{O}(75: 25)$ as the eluent to afford chaetomugilin $\mathrm{J}(2,7.7 \mathrm{mg})$.

\section{Cell Lines and Cell Culture}

Ovarian cancer cell A2780 was purchased from the Chinese Academy of Medical Sciences (Beijing, China), the wildtype HEK293T cell line and the HEK293T cell line which was overexpressed parkin were provide to us by the Tumor Biotherapy Center of Jilin Province People's Hospital. In this institution, the HEK293T cell lines which cultured by the team of professor Xiaodan $\mathrm{Lu}$ were purchased from genechem biotechnology company, Shanghai. The A2780 cell was grown in RPMI-1640 (Gibco Life Technologies, Carlsbad, CA) and HEK293T cell was grown in DMEM (Gibco Life Technologies, Carlsbad, CA). Both the mediums 
were supplemented with $10 \%$ fetal bovine serum (Invitrogen, Carlsbad, CA) at $37^{\circ} \mathrm{C}, 5 \% \mathrm{CO}_{2}$ concentration.

\section{Reagents and Antibodies}

MTT[3-(4,5-Dimethyl-2-thiazolyl)-2,5-diphenyl-2H-tetrazolium bromide] and cisplatin were purchased from Sigma-Aldrich (St. Louis, MO, USA). The antibodies used in this study including: antibodies against bcl-2, bax, bak, were purchased from Abcam (Cambridge, MA). Antibodies against cleaved caspase 3, mcl-1, pink1, parkin, beta-actin, HRP-linked secondary antibody were purchased from Proteintech (Chicago, IL). Antibodies against grp78, chop were purchased from Santa Cruz Biotechnology (Santa Cruz, CA).

\section{Cell Viability Assay}

A2780 cells were seeded in 96-well plates at a density of 7000 cells/well. After $24 \mathrm{~h}$, cell viability was measured using MTT assay and absorbance values were measured at $490 \mathrm{~nm}$ using a Vmax Microplate Reader (Molecular Devices, LLC, Sunnyvale, CA).

\section{Western Blot Analysis}

A2780 cells were treated with compounds according to experimental requirements. Then cells were lysed in cell lysis buffer and incubated on ice for $30 \mathrm{~min}$. The lysates were centrifuged at $12000 \mathrm{~g}$ for $10 \mathrm{~min}$ at $4^{\circ} \mathrm{C}$, the protein levels of supernatant were quantified with a bicinchoninic acid (BCA) kit (Thermo Fisher Scientific, USA) according to the manufacturer's instructions. Proteins were separated on dodecyl sulfate, sodium salt-polyacrylamide gel electrophoresis (SDS-PAGE), transferred on poly-vinylidene fluoride (PVDF) membranes, and blocked with 5\% skim milk for $2 \mathrm{~h}$. The membranes were then probed with primary antibodies overnight at $4^{\circ} \mathrm{C}$, followed by secondary antibody for $2 \mathrm{~h}$ at room temperature. Electrochemical luminescence (ECL) reagent (Thermo Fisher Scientific, Rockford, IL) was used for immune-detection and visualization using Syngene Bio Imaging (Synoptics, Cambridge, UK).

\section{Flow Cytometry}

Cells were seeded in 6-well plates at a density of $2 \times 10^{5}$ cells/ well overnight. Then the cells were exposed to different indicated conditions. Annexin V- FITC/PI staining (BD Biosciences, San Diego, CA) was used to measure cellular apoptosis. JC-1, which chemical name is 5.5',6,6'-Tetrachloro -1,1',3,3'-tetraethyl-imidacarbocyanine iodide (Beyotime Biotechnology, Shanghai, China) was used to detect mitochondrial membrane potential (MMP). The production of ROS was evaluated by 2 ', 7 ' - dichlorofluorescein diacetate (DCFH-DA) (Beyotime Biotechnology, Shanghai, China). Then the cells were analyzed by guava easycyte flow cytometry (guava easycyte, MA, USA).

\section{Measurement of Mitochondrial ROS}

The levels of mitochondrial ROS (MitoROS) were detected by MitoSOX Red indicator (Thermo Fisher, Scientific, USA). A2780 cells were seeded in 24-well plates at a density of $4 \times 10^{4}$ cells/well overnight. They were treated with compounds according to experimental requirements. After treatment, cells were stained with $5 \mu \mathrm{M}$ Mitosox for $20 \mathrm{~min}$ at $37^{\circ} \mathrm{C}$. After washing with PBS, the cells were observed using ZEISS (Imager Z2) laser microscope.

\section{Colony Formation Assay}

Cells were seeded at 500 cells per well in 24-well plates overnight. Then cells were treated with compounds as indicated by the experiment, and they were allowed to continue growing. Plates were placed in an incubator and left them there until cells in control dishes have formed sufficiently large clones. Then the medium was removed, and cells were washed in PBS once, fixed and stained in a mixture of $6 \%$ glutaraldehyde and $0.5 \%$ crystal violet for $2 \mathrm{~h}$. The mixture of glutaraldehyde and crystal violet was removed and cells were rinsed several times with water. The numbers of colonies were counted by software Image J.

\section{Statistical Analysis}

All experimental data represent at least 3 independent experiments and the results were represented as mean values \pm standard deviation (S.D). $P<0.05$ were considered statistically significant difference and $P<0.01$ was considered extremely significant. Statistical analysis was performed with GraphPad Prism 7.0 (La Jolla, CA).

\section{Results}

\section{Chaetomugilin J Enhances the Apoptosis Induced by Cisplatin in A2780 Ovarian Cancer Cells}

First, we identified the compound (Figure 1A). The compound is characterized as follows:

$\mathrm{H}^{1}$-NMR $\left(600 \mathrm{MHz}, \mathrm{CDCl}_{3}\right): \delta: 7.47(1 \mathrm{H}, \mathrm{s}, \mathrm{H}-1), 6.45$ $(1 \mathrm{H}, \mathrm{s}, \mathrm{H}-4), 3.48(1 \mathrm{H}, \mathrm{dd}, \mathrm{J}=10.2,2.2 \mathrm{~Hz}, \mathrm{H}-8), 6.02(1 \mathrm{H}, \mathrm{d}$, $\mathrm{J}=15.5 \mathrm{~Hz}, \mathrm{H}-9), 6.48(1 \mathrm{H}, \mathrm{dd}, \mathrm{J}=15.5,6.5 \mathrm{~Hz}, \mathrm{H}-10), 2.24$ 
A<smiles>C/C=C(\C)C(=O)C[C@H](O)[C@@H](O)C(=O)Cl</smiles>

C

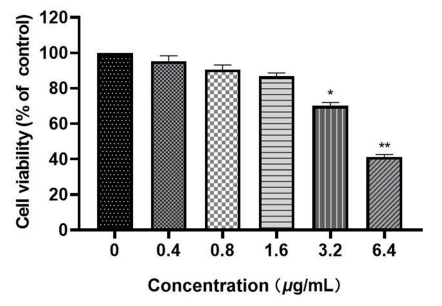

E

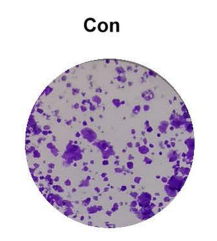

F

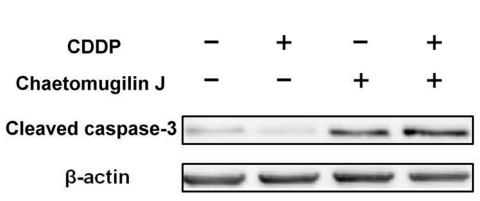

H

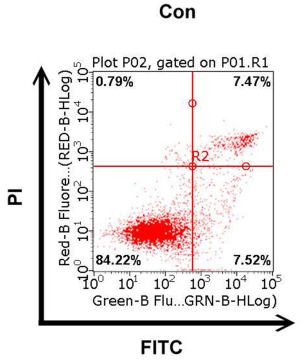

B

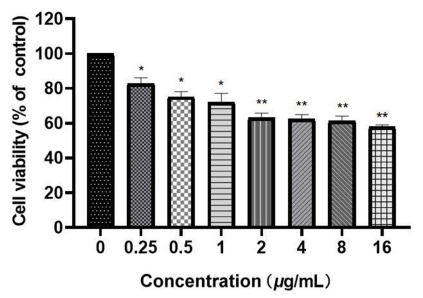

D
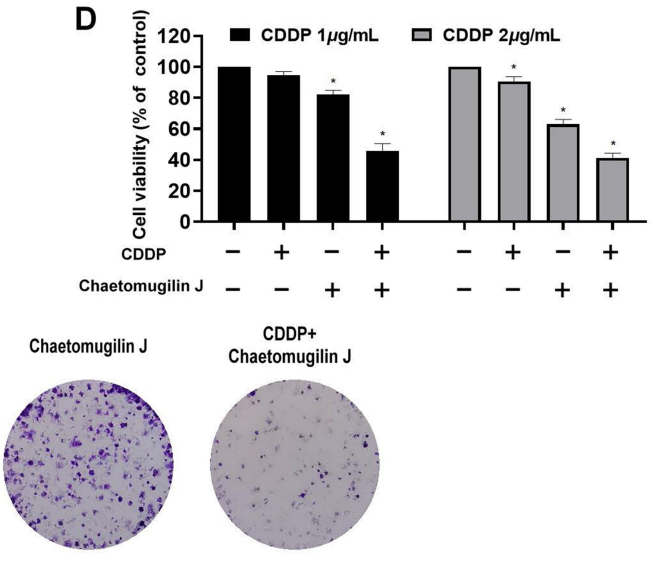

G

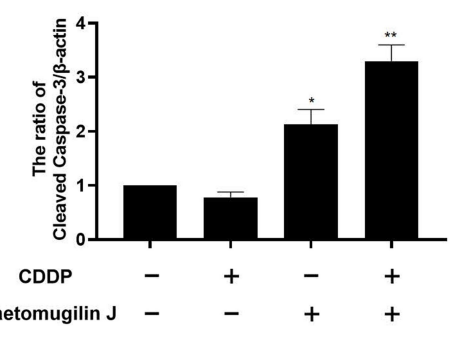

Chaetomugilin J

CDDP+Chaetomugilin J
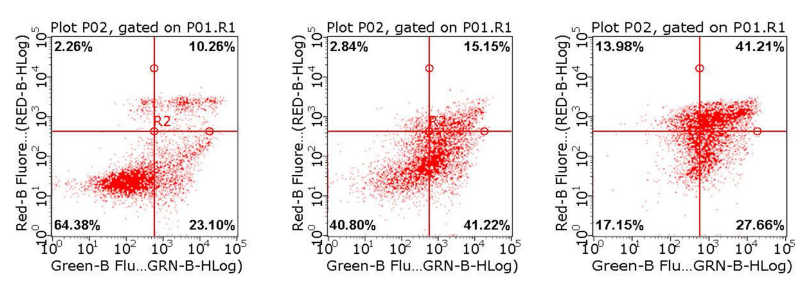

Figure I Chaetomugilin J enhances the apoptosis induced by cisplatin in ovarian cancer cells A2780. (A) The chemical structure of chaetomugilin J. (B) Cells were treated with $0-16 \mu \mathrm{g} / \mathrm{mL}$ chaetomugilin J for $24 \mathrm{~h}$, cell viability was measured by MTT assay. (C) Cells were treated with 0-6.4 $\mu \mathrm{g} / \mathrm{mL}$ cisplatin (CDDP) for $24 \mathrm{~h}$, cell viability was measured by MTT assay. (D) Cells were treated with $0.25 \mu \mathrm{g} / \mathrm{mL}$ chaetomugilin J combined with I, $2 \mu \mathrm{g} / \mathrm{mL}$ cisplatin for $24 \mathrm{~h}$, cell viability was measured by MTT assay. (E) Cells were treated with $0.25 \mu \mathrm{g} / \mathrm{mL}$ chaetomugilin J, I $\mu \mathrm{g} / \mathrm{mL}$ cisplatin alone or combination for 8 days, the clone formation was detected by colony formation assay. (F and G) Cells were treated with $0.25 \mu \mathrm{g} / \mathrm{mL}$ chaetomugilin J, $1 \mu \mathrm{g} / \mathrm{mL}$ cisplatin alone or combination for $24 \mathrm{~h}$, the expression of Cleaved-caspase 3 was measured by Western blot. (H) Cells were treated with $0.25 \mu \mathrm{g} / \mathrm{mL}$ chaetomugilin J, I $\mu \mathrm{g} / \mathrm{mL}$ cisplatin alone or combination for $24 \mathrm{~h}$, the apoptotic rate was measured by flow cytometry. Data are presented as mean $\pm \mathrm{SD}, \mathrm{n}=3 . * \mathrm{P}<0.05$, $* * P<0.01$.

$(1 \mathrm{H}$, sept, $\mathrm{J}=6.5 \mathrm{~Hz}, \mathrm{H}-11), 1.42(1 \mathrm{H}, \mathrm{qd}, \mathrm{J}=7.5,6.5 \mathrm{~Hz}$, $\mathrm{H}-12), 0.89(3 \mathrm{H}, \mathrm{t}, \mathrm{J}=7.5 \mathrm{~Hz}, \mathrm{H}-13), 1.33\left(3 \mathrm{H}, \mathrm{s}, 7-\mathrm{CH}_{3}\right), 1.07$ $\left(3 \mathrm{H}, \mathrm{d}, \mathrm{J}=6.5,11-\mathrm{CH}_{3}\right), 2.62\left(1 \mathrm{H}, \mathrm{dd}, \mathrm{J}=17.3,10.2, \mathrm{H}-1^{\prime} \mathrm{A}\right)$, $3.28\left(1 \mathrm{H}, \mathrm{dd}, \mathrm{J}=17.3,2.2, \mathrm{H}-1^{\prime} \mathrm{B}\right), 6.66\left(1 \mathrm{H}, \mathrm{q}, \mathrm{J}=7.1, \mathrm{H}-4^{\prime}\right)$, $1.80\left(3 \mathrm{H}, \mathrm{d}, \mathrm{J}=7.1, \mathrm{H}-5^{\prime}\right), 1.73\left(3 \mathrm{H}, \mathrm{s}, 3^{\prime}-\mathrm{CH}_{3}\right)$.
$\mathrm{C}^{13}$-NMR (500 MHz, $\left.\mathrm{CDCl}_{3}\right): \delta: 145.72(\mathrm{~d}, \mathrm{C}-1)$, 157.17 (s, C-3), 104.51 (d, C-4), 141.79 (s, C-4a), 106.47 (s, C-5), 191.82 (s, C-6), 74.07 (s, C-7), 40.51 (d, C-8), 119.50 (s, C-8a), 120.35 (d, C-9), 146.12 (d, C-10), 38.79 (d, C-11), 29.16 (t, C-12), 11.67 (q, C-13), 
$26.79\left(\mathrm{q}, 7-\mathrm{CH}_{3}\right), 19.38\left(\mathrm{q}, 11-\mathrm{CH}_{3}\right), 38.46(\mathrm{t}, \mathrm{C}-1)$, 199.41 (s, C-2'), 137.96 (d, C-4'), 14.69 (q, C-5'), 10.99 (q, 3'- $\mathrm{CH}_{3}$ ).

HRESIMS: m/z, $391.1662(\mathrm{M}+\mathrm{H}){ }^{+}$, (cal: 391.1671), formula: $\mathrm{C}_{22} \mathrm{H}_{27} \mathrm{ClO}_{4}$. This compound was firstly reported from a marine-fish-derived Chaetomium species. ${ }^{6} \mathrm{We}$ also isolated this compound from a plant endophytic fungus Chaetomium globosum. ${ }^{21}$

Next, to measure the viability of A2780 cells treated with chaetomugilin $\mathrm{J}$ alone or combined with cisplatin, we treated A2780 cells with different concentrations of chaetomugilin $\mathrm{J}(0-16 \mu \mathrm{g} / \mathrm{mL})$ or cisplatin $(0-6.4 \mu \mathrm{g} / \mathrm{mL})$ for $24 \mathrm{~h}$, and MTT assay was used to detect the cell viability. As shown in Figure 1B and C, chaetomugilin J inhibited the viability of A2780 cells in a dose-dependent manner, while cisplatin showed no significant inhibitory effect on cell viability at low concentrations (0-3.2 $\mu \mathrm{g} / \mathrm{mL})$. Subsequently, cells were treated with $0.25 \mu \mathrm{g} / \mathrm{mL}$ chaetomugilin $\mathrm{J}$ combined with 1 $\mu \mathrm{g} / \mathrm{mL}$ or $2 \mu \mathrm{g} / \mathrm{mL}$ cisplatin, cell viability and proliferation were detected by MTT and colony formation assays. Chaetomugilin J significantly reduced the cell viability and number of colonies in A2780 cells when combined with low-dose cisplatin (Figure 1D and E).

Next, we examined whether or not chaetomugilin J could induce the apoptosis in A2780 cells or increase the apoptosis combined with cisplatin. A2780 cells were treated with chaetomugilin $\mathrm{J}$ alone or combined with cisplatin for $24 \mathrm{~h}$, and the expression of cleaved caspase- 3 was detected by Western blot. Chaetomugilin J combined with cisplatin significantly increased the expression of cleaved caspase-3 (Figure 1F and G). Subsequently, we detected the apoptosis rate of cells treated with chaetomugilin $\mathrm{J}$ alone or combined with cisplatin for $24 \mathrm{~h}$ by Annexin V-FITC/PI staining using flow cytometry. Results showed that chaetomugilin J could activate apoptosis, and chaetomugilin $\mathrm{J}$ combined with cisplatin significantly increased the apoptosis rate of cells (Figure 1H, Table S1).

\section{The Apoptosis Induced by Chaetomugilin J Combined with Cisplatin is Independent of the Endoplasmic Reticulum Stress Pathway}

The endoplasmic reticulum stress is a main pathway leading to cell apoptosis. ${ }^{14}$ When the misfolded proteins increase significantly, the endoplasmic reticulum stress is activated. ${ }^{22}$ We detected the expression of the chaperon protein grp78, which is activated under endoplasmic reticulum stress. Cells were treated with $0.25 \mu \mathrm{g} / \mathrm{mL}$ chaetomugilin $\mathrm{J}, 1 \mu \mathrm{g} / \mathrm{mL}$ cisplatin, or both compounds. As shown in Figure 2, the expression of grp78 in A2780 cells decreased significantly when cells were treated with chaetomugilin J combined with cisplatin for $6 \mathrm{~h}$. In addition, chop is a transcription factor activated by the endoplasmic reticulum stress and is involved in regulating the endoplasmic reticulum stress apoptosis pathway. ${ }^{10}$ Western blot showed that the expression of chop decreased when cells were treated with chaetomugilin J combined with cisplatin. This indicated that the apoptosis of A2780 cells induced by chaetomugilin $\mathrm{J}$ combined with cisplatin was independent of the endoplasmic reticulum stress pathway.

\section{Chaetomugilin J Combined with Cisplatin Induces Mitochondrial Dysfunction}

Mitochondria plays a crucial role in regulating cell apoptosis. ${ }^{23}$ To further evaluate whether or not chaetomugilin

A

B

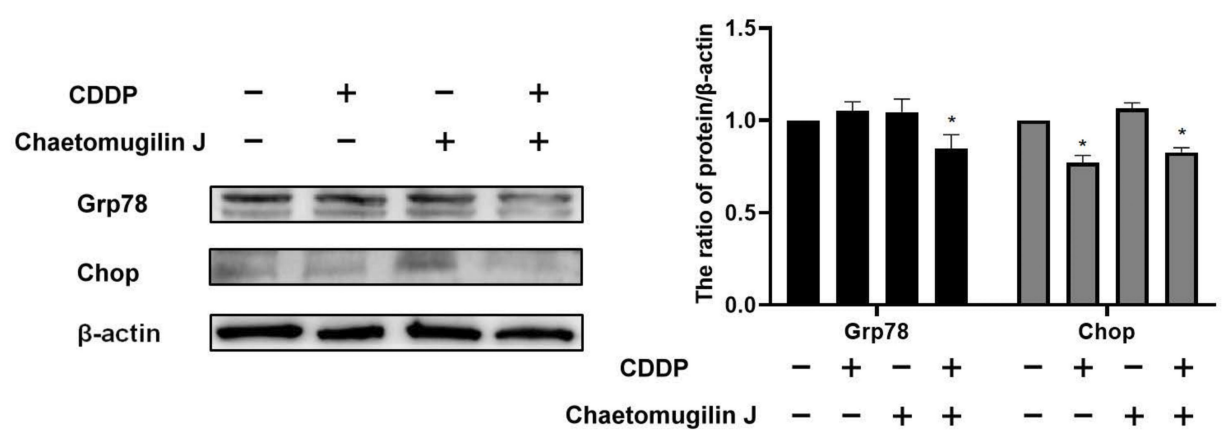

Figure 2 The apoptosis of A2780 cells induces by chaetomugilin J combines with cisplatin is independent of the endoplasmic reticulum stress pathway. (A and B) Cells were treated with $0.25 \mu \mathrm{g} / \mathrm{mL}$ chaetomugilin J, I $\mu \mathrm{g} / \mathrm{mL}$ cisplatin alone or combination for $6 \mathrm{~h}$, the expressions of Grp78 and Chop were measured by Western blot. Data are presented as mean $\pm S D, n=3$. $* P<0.05$. 
J combined with cisplatin could target mitochondria and impair its functions, we evaluated mitochondrial functions as follows. The levels of intracellular ROS and mitochondrial ROS were detected using DCFH-DA or MitoSOX staining. As shown in Figure 3A and B and Table S1, the intracellular
ROS and mitochondrial ROS levels of the cells treated with chaetomugilin $\mathrm{J}$ combined with cisplatin were significantly higher than those in the control group or other treated groups. This indicated that the combination of the two compounds induced an increase in ROS levels in ovarian cancer A2780

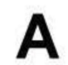

B

Con

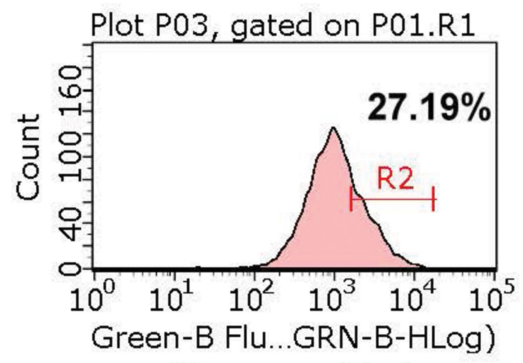

Chaetomugilin $\mathrm{J}$

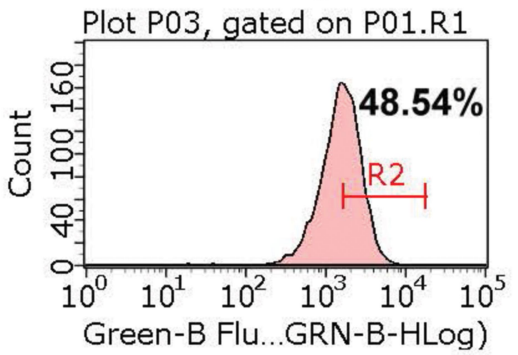

Con

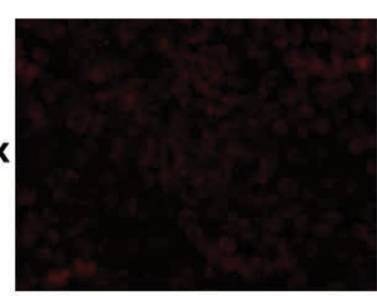

Con

CDDP
CDDP

Plot P03, gated on P01.R1

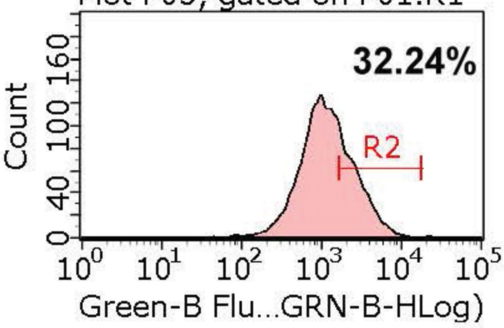

Chaetomugilin J+CDDP

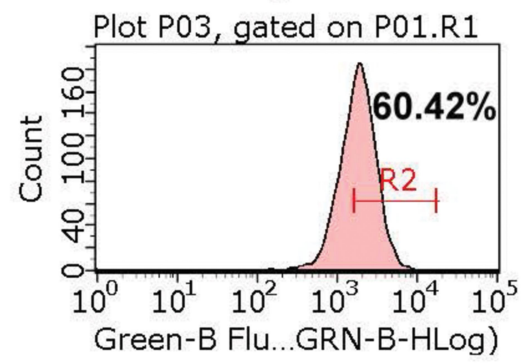

Chaetomugilin J

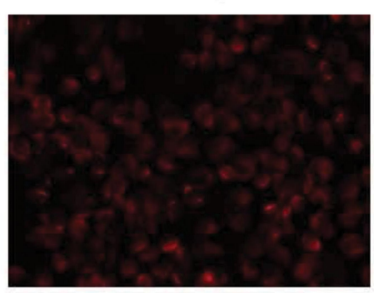

Chaetomugilin J
Chaetomugilin J

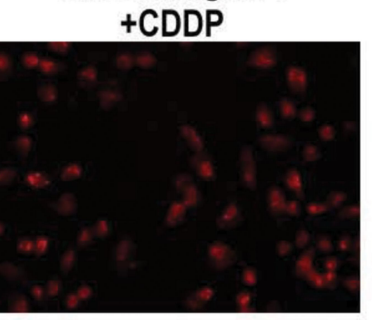

Chaetomugilin J

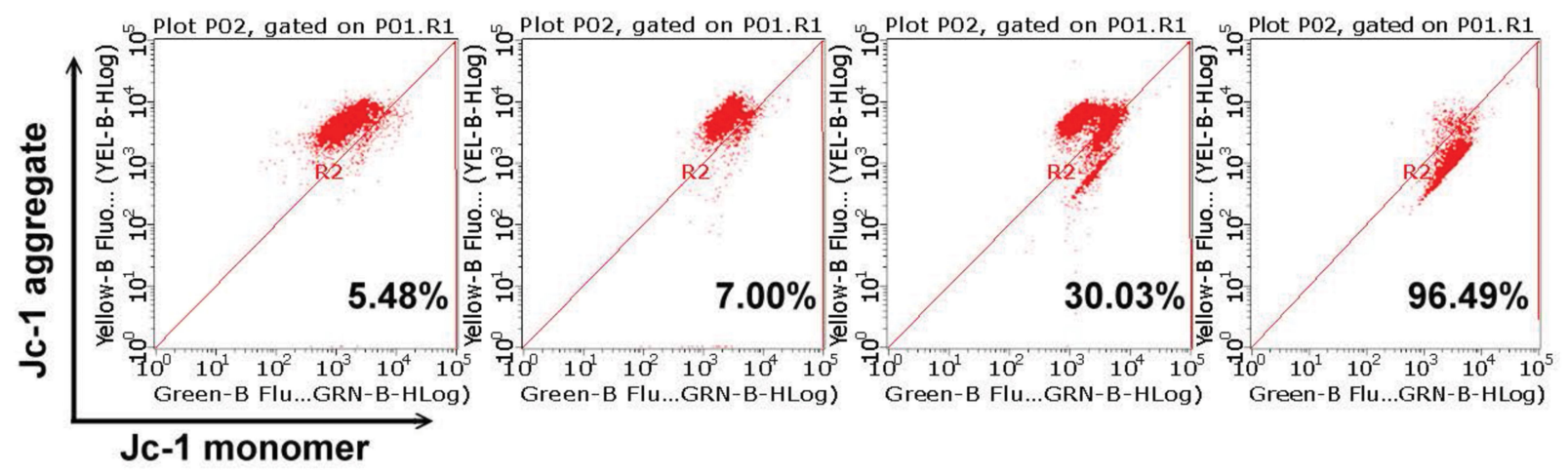

Figure 3 Chaetomugilin J combines with cisplatin further induces mitochondrial dysfunction in ovarian cancer A2780 cells. (A) Cells were treated with $0.25 \mu$ g/mL chaetomugilin J, I $\mu \mathrm{g} / \mathrm{mL}$ cisplatin alone or combination for $24 \mathrm{~h}$, the levels of intracellular ROS were detected using DCFH-DA by flow cytometry. (B) Cells were treated with $0.25 \mu \mathrm{g} / \mathrm{mL}$ chaetomugilin J, I $\mu \mathrm{g} / \mathrm{mL}$ cisplatin alone or combination $24 \mathrm{~h}$, the levels of mitochondrial ROS were detected using MitoSox Red mitochondrial superoxide indicator by fluorescence microscope. (C) Cells were treated with $0.25 \mu \mathrm{g} / \mathrm{mL}$ chaetomugilin J, I $\mu \mathrm{g} / \mathrm{mL}$ cisplatin alone or combination for $24 \mathrm{~h}$, the mitochondrial membrane potential with JC-I by flow cytometry. Data are presented as mean \pm SD, $n=3$. 
cells, which might impair mitochondrial function through the increase in ROS and induce mitochondrial pathway apoptosis.

The normal mitochondrial membrane potential is necessary for maintaining oxidative phosphorylation and ATP production and essential for maintaining mitochondrial function. ${ }^{24,25}$ Mitochondrial membrane potential decreases in apoptosis. As shown in Figure $3 \mathrm{C}$ and Table S1, chaetomugilin $\mathrm{J}$ decreased the JC-1 aggregate and increased the JC-1 monomer in A2780 cells, while chaetomugilin $\mathrm{J}$ combined with cisplatin treatment could more significantly induce this phenomenon. This suggested that the combination treatment could further impair mitochondrial function.

\section{Chaetomugilin J Combined with Cisplatin Enhances Mitochondrial Pathway}

\section{Apoptosis}

We investigated whether or not chaetomugilin J combined with cisplatin-activated mitochondrial pathway apoptosis by detecting the expression of bcl-2 family-related proteins. As shown in Figure 4, the bak/mcl-1 and bax/bcl-2 ratios increased when A2780 cells were treated with chaetomugilin $\mathrm{J}$ combined with cisplatin. This indicated that chaetomugilin $\mathrm{J}$ combined with cisplatin-enhanced mitochondrial pathway apoptosis in ovarian cancer A2780 cells.

\section{Chaetomugilin J Inhibits Mitophagy Induced by Cisplatin}

Mitophagy is a type of autophagy that eliminates redundant or damaged mitochondria. ${ }^{26}$ It plays an important role in mitochondrial function. The expression of pink1 and parkin, which are mitophagy-related proteins, were detected to reflect mitophagy. When A2780 cells were treated with chaetomugilin J combined with cisplatin for $6 \mathrm{~h}$, the expressions of pink1 and parkin decreased (Figure 5A and B). These results indicated that the mitophagy of A2780 cells were inhibited when cells were treated with chaetomugilin $\mathrm{J}$ combined with cisplatin.

\section{Overexpression of Parkin Attenuates Apoptosis Induced by Chetomugilin J Combined with Cisplatin}

In the aforementioned results, chaetomugilin $\mathrm{J}$ combined with cisplatin inhibited pink1/parkin-mediated mitophagy in A2780 cells. Therefore, we wanted to investigate the role of mitophagy in apoptosis using the HEK293T cell line. First, we detected the expression of parkin to ensure the efficiency of overexpression. The expression of parkin was much higher in overexpressed cells than in wild-type cells, and chaetomugilin $\mathrm{J}$ combined with cisplatin decreased the expression of parkin cells (Figure 6C). In the cells in which parkin was overexpressed, the cell viability and number of colonies were increased (Figure 6A and B), and bax/bcl-2 ratio was decreased (Figure $6 \mathrm{D}$ and $\mathrm{E}$ ) when cells were treated with chaetomugilin J combined with cisplatin. In addition, when cells were treated with inhibitors of mitophagy, Mdivi-1, cell viability decreased and apoptosis rate increased (Figure 6F and G). The overexpression of parkin attenuated the apoptosis induced by chaetomugilin $\mathrm{J}$ combined with cisplatin, and inhibition of mitophagy resulted in increased apoptosis.

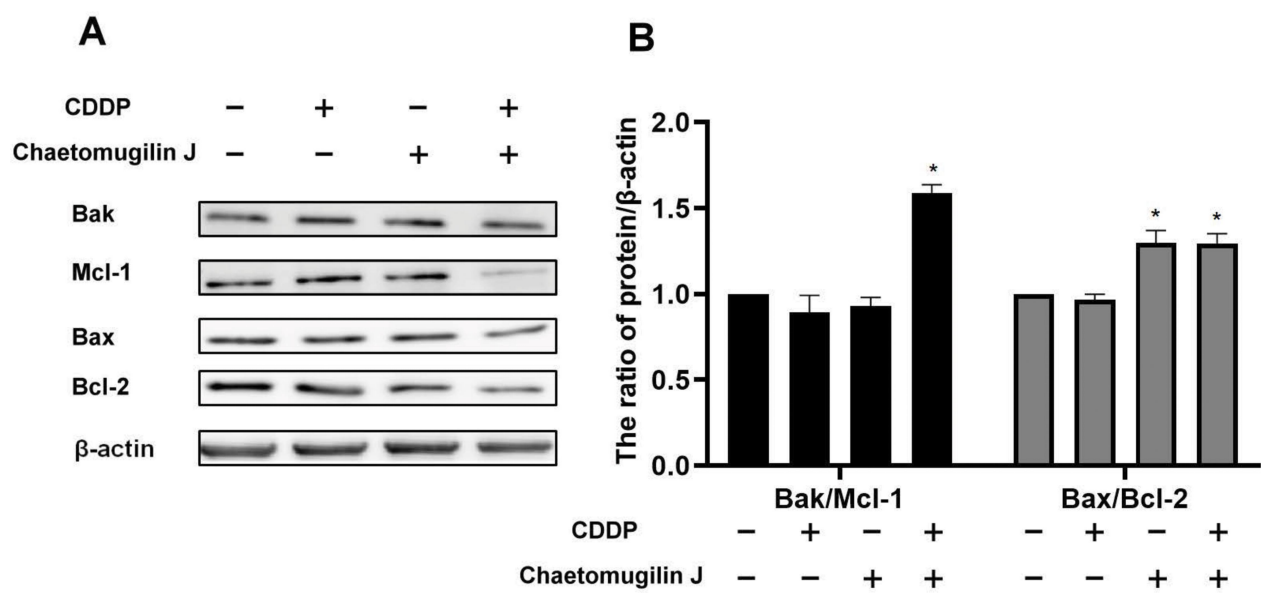

Figure 4 Chaetomugilin J combines with cisplatin enhances the mitochondrial pathway apoptosis of ovarian cancer A2780 cells. (A and B) Cells were treated with $0.25 \mu$ g/ $\mathrm{mL}$ chaetomugilin J, I $\mu \mathrm{g} / \mathrm{mL}$ cisplatin alone or combination for $24 \mathrm{~h}$, the ratios of Bak/Mcl-I and Bax/Bcl-2 were measured by Western blot. Data are presented as mean \pm SD, $\mathrm{n}=3 . * P<0.05$. 

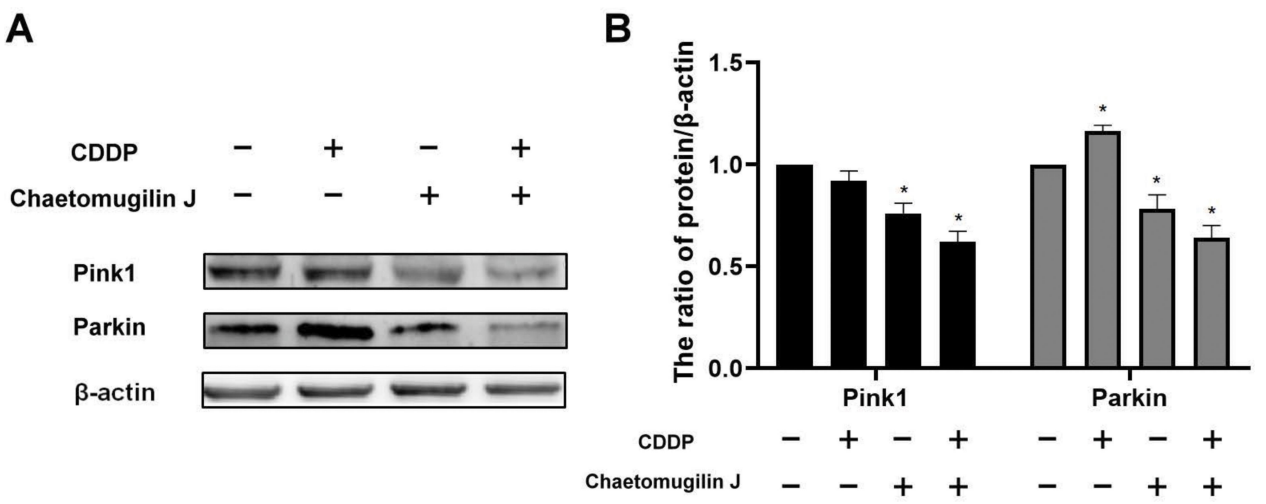

Figure 5 Chaetomugilin J inhibited mitophagy induced by cisplatin. (A and B) Chaetomugilin J inhibited mitophagy induced by cisplatin. Cells were treated with $0.25 \mu \mathrm{g} / \mathrm{mL}$ chaetomugilin J, I $\mu \mathrm{g} / \mathrm{mL}$ cisplatin alone or combination for $6 \mathrm{~h}$, the expressions of Pink I, Parkin were measured by Western blot. Data are presented as mean \pm SD, $\mathrm{n}=3$. $* P<$ 0.05 .

\section{Discussion}

It is believed that the avoidance of cancer apoptosis is related to chemoresistance. ${ }^{9,27}$ Therefore, it is necessary to pay more attention to new antitumor drugs or compounds that increase cisplatin sensitivity in ovarian cancer cells. Natural compounds exhibit antitumor activity and can potentially reverse chemoresistance in a variety of cancers. ${ }^{4,28}$ In this study, it was found that chaetomugilin $\mathrm{J}$ significantly reduced cell viability and induced apoptosis in ovarian cancer A2780 cells. As is known, there are some toxic and adverse effects on patients when highdose cisplatin is used in the clinic. ${ }^{29}$ However in our research, it is worth noting that the MTT assay showed that a low dose of chaetomugilin $J$ significantly reduced cell viability, but the inhibition efficiency of cell viability was not dose-dependent. This suggested that chaetomugilin $\mathrm{J}$ might activate cell death by triggering it directly rather than via toxicity, as it also has the advantages of low toxicity and high anti-tumor activity.

Our findings showed that, $0.25 \mu \mathrm{g} / \mathrm{mL}$ chaetomugilin J combined with $1 \mu \mathrm{g} / \mathrm{mL}$ cisplatin had an obvious synergistic effect (CI: 0.20, FigureS1). DCFH-DAstaining flow cytometry indicated that the concentration of reactive oxygen species (ROS) increased significantly (60.42\%, Table S1). In addition, JC-1-staining flow cytometry showed that MMP was decreased significantly (96.49\%, Table S1) and Annexin V-FITC/PI-staining flow cytometry revealed a significant increase in apoptosis rate $(82.82 \%$, Table S1) when cells were treated with $0.25 \mu \mathrm{g} / \mathrm{mL}$ chaetomugilin $\mathrm{J}$ combined with $1 \mu \mathrm{g} / \mathrm{mL}$ cisplatin. Therefore, we focused on the mechanism of apoptosis induced by chaetomugilin $\mathrm{J}$ combined with low-dose cisplatin in ovarian cancer cells.
In cancer cells, the endoplasmic reticulum and mitochondria are important organelles involved in chemoresistance. Endoplasmic reticulum stress is a common cellular stress response that is brought about by a variety of conditions that disturb cellular homeostasis, such as the accumulation of misfolded or unfolded proteins. ${ }^{13}$ These defective proteins accumulate in the ER lumen and may result in cell damage, one of the pathways that induce apoptosis. In the present study, the expression of chop and grp78 was decreased, which indicated that endoplasmic reticulum stress was not activated. It could be that the accumulated proteins are partially degraded through autophagy, therefore attenuating the stress of the endoplasmic reticulum lumen.

Mitochondria play a crucial role in regulating cell death. Normal mitochondrial functions are crucial for maintaining cell survival, growth, proliferation, and metastasis. ${ }^{30,31}$ Under stress conditions, mitochondria are impaired and dysfunctional mitochondria lead to enhanced production of ROS, and extensive cell damage, promoting apoptosis. Additionally, clearance of damaged mitochondria via mitophagy may have a protective effect on cells. $^{32,33}$ In our study, chaetomugilin $\mathrm{J}$ combined with cisplatin could target mitochondria, increase ROS, and impair mitochondrial function. We also found that the apoptosis of cells was decreased when cells overexpressed parkin, confirming the protective effect of mitophagy on cells. However, chaetomugilin J combined with cisplatin could inhibit pink1/parkin-mediated mitophagy, therefore weakening the protective effect of mitophagy. This was the main mechanism by which chaetomugilin J induced apoptosis and increased sensitivity to cisplatin.

In summary, we evaluated the antitumor activity of chaetomugilin $\mathrm{J}$ and the mechanism of apoptosis induced by 

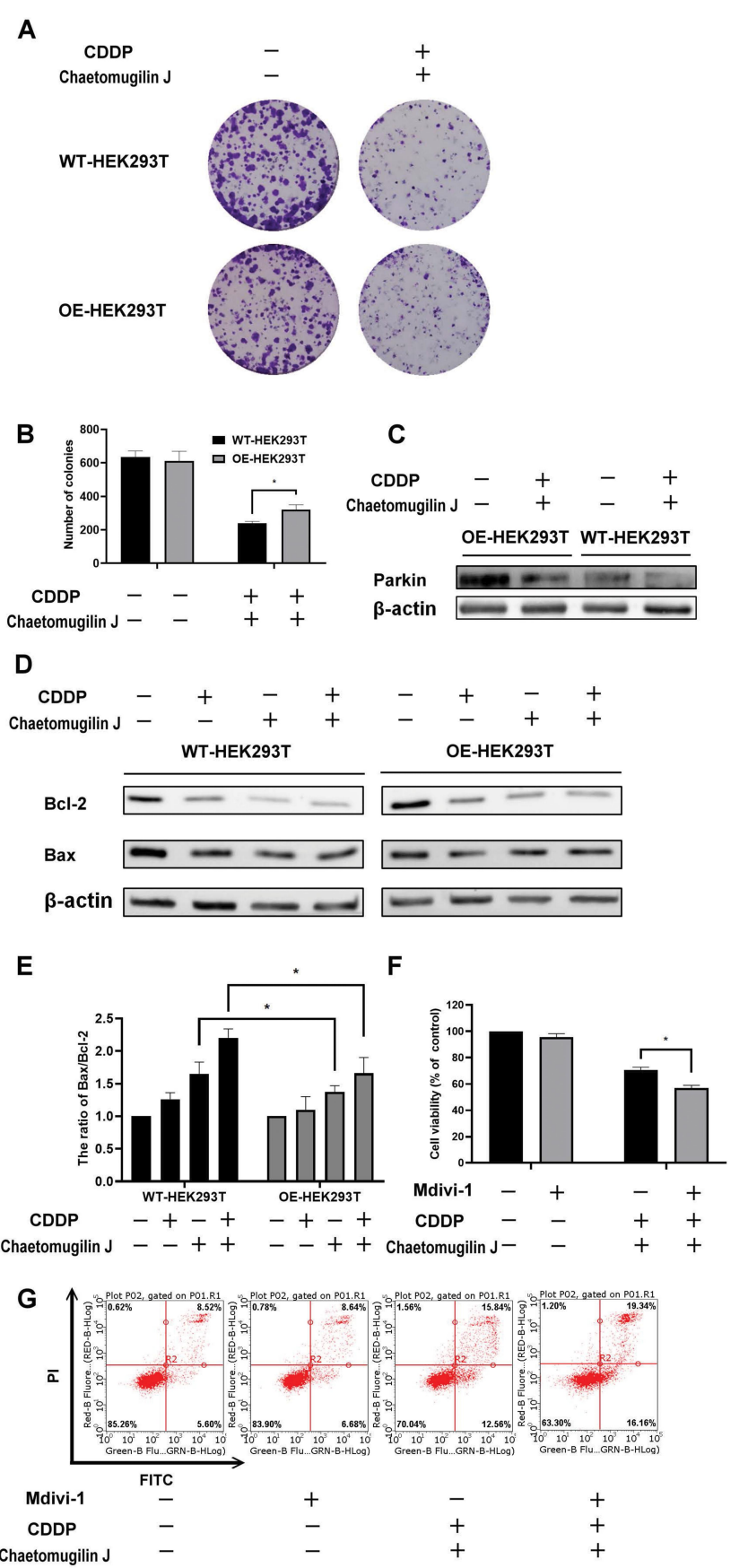

Figure 6 Overexpression of parkin attenuates apoptosis induced by chaetomugilin $\mathrm{J}$ combined with cisplatin. (A and B). Cells were treated with $0.25 \mu \mathrm{g} / \mathrm{mL}$ chaetomugilin $\mathrm{J}, \mathrm{I} \mu \mathrm{g} / \mathrm{mL}$ cisplatin combination with two drugs for $24 \mathrm{~h}$, the clone formation was detected by colony formation assay. (C) The expression efficiency of Parkin in wildtype or overexpressed Parkin cells was measured by Western blot. (D and E) Cells were treated with $0.25 \mu \mathrm{g} / \mathrm{mL}$ chaetomugilin J, I $\mu \mathrm{g} / \mathrm{mL}$ cisplatin alone or combination for $6 \mathrm{~h}$, the expressions of $\mathrm{Bax} / \mathrm{Bcl}-2$ were measured by Western blot. (F) HEK293T cells were treated with Mdivi-I alone or combined with chaetomugilin J and cisplatin, cell viability was measured by MTT assay. (G) HEK293T cells were treated with Mdivi-I alone or combined with chaetomugilin J and cisplatin, the apoptotic rate was measured by flow cytometry. Data are presented as mean $\pm S D, n=3$. $* P<0.05$.

chaetomugilin J combined with low-dose cisplatin in A2780 cells. We found out that chaetomugilin J combined with lowdose cisplatin significantly impaired mitochondrial function, which is manifested by decreased mitochondrial membrane potentials and increased ROS levels. At the same time, the combination of the two compounds weakened the protective effect of mitophagy on cells by inhibiting mitophagy. We first detected the apoptosis mechanism of ovarian cancer cells induced by chaetomugilin $\mathrm{J}$ alone or in combination with low-dose cisplatin. We suggest that this natural compound not only has antitumor activity, but also increases the sensitivity of ovarian cancer cells to cisplatin by targeting mitochondria. In addition, chaetomugilin $\mathrm{J}$ could also help decrease the dose of platinum chemotherapy drugs during treatment.

\section{Funding}

This study was funded by the National Natural Science Foundation of China (81772794, 81672948, 81472419, 31870332, 31470414); Jilin Provincial Industrial Innovation Project (2018C052-7); Jilin Provincial Research Foundation for the Development of Science and Technology Projects (20191004004TC); Natural Science Foundation of Jilin Province (No. 20200703010ZP, 20191008010TC); the 13th Five-year Science and Technology Project of Jilin Provincial Education Department (JJKH20180190KJ); the Ability Establishment of Sustainable Use for Valuable Chinese Medicine resources (2060302), and the Fundamental Research Funds for the Central Universities, JLU.

\section{Disclosure}

The authors report no conflicts of interest for this work.

\section{References}

1. Ozols RF, Bookman MA, Connolly DC, et al. Focus on epithelial ovarian cancer. Cancer Cell. 2004;5(1):19-24. doi:10.1016/S15356108(04)00002-9

2. Modesitt SC, Jazaeri AA. Recurrent epithelial ovarian cancer: pharmacotherapy and novel therapeutics. Expert Opin Pharmacother. 2007;8(14):2293-2305. doi:10.1517/14656566.8.14.2293

3. Norouzi-Barough L, Sarookhani MR, Sharifi M, Moghbelinejad S, Jangjoo S, Salehi R. Molecular mechanisms of drug resistance in ovarian cancer. J Cell Physiol. 2018;233(6):4546-4562. doi:10.1002/ jcp.26289

4. Nobili S, Lippi D, Witort E, et al. Natural compounds for cancer treatment and prevention. Pharmacol Res. 2009;59(6):365-378. doi:10.1016/j.phrs.2009.01.017

5. Yang S, Qin J, et al. Extraction of flavonoids from Cyclocarya paliurus (Juglandaceae) leaves using ethanol/salt aqueous two-phase system coupled with ultrasonic. J Food Process Pres. 2020;44(6):284-293. doi:10.1111/jfpp.14469

6. Muroga Y, Yamada. T, et al. Chaetomugilins I-O, new potent cytotoxic metabolites from a marine-fish-derived Chaetomium species. Stereochem Biol Activities Tetrahedron. 2009;65(36):7580-7586. 
7. Yasuhide M, Yamada T, Numata A, Tanaka R. Chaetomugilins, new selectively cytotoxic metabolites, produced by a marine fish-derived Chaetomium species. J Antibiot. 2008;61(10):615-622. doi:10.1038/ ja.2008.81

8. Coukos G, Rubin SC. Chemotherapy resistance in ovarian cancer: new molecular perspectives. Obstet Gynecol. 1998;91(5 Pt 1):783-792.

9. Delbridge AR, Strasser A. The BCL-2 protein family, BH3-mimetics and cancer therapy. Cell Death Differ. 2015;22(7):1071-1080. doi: $10.1038 / \mathrm{cdd} .2015 .50$

10. Iurlaro R, Munoz-Pinedo C. Cell death induced by endoplasmic reticulum stress. FEBS J. 2016;283(14):2640-2652. doi:10.1111/ febs. 13598

11. Shen X, Zhang K, Kaufman RJ. The unfolded protein response-a stress signaling pathway of the endoplasmic reticulum. J Chem Neuroanat. 2004;28(12):79-92. doi:10.1016/j.jchemneu.2004.02.006

12. Tabas I, Ron D. Integrating the mechanisms of apoptosis induced by endoplasmic reticulum stress. Nat Cell Biol. 2011;13(3):184-190. doi:10.1038/ncb0311-184

13. Xu Y, Wang C, Li Z. A new strategy of promoting cisplatin chemotherapeutic efficiency by targeting endoplasmic reticulum stress. Mol Clin Oncol. 2014;2(1):3-7. doi:10.3892/mco.2013.202

14. Xu Y, Yu H, Qin H, et al. Inhibition of autophagy enhances cisplatin cytotoxicity through endoplasmic reticulum stress in human cervical cancer cells. Cancer Lett. 2012;314(2):232-243. doi:10.1016/j. canlet.2011.09.034

15. Vakifahmetoglu-Norberg H, Ouchida AT, Norberg E. The role of mitochondria in metabolism and cell death. Biochem Biophys Res Commun. 2017;482(3):426-431. doi:10.1016/j.bbrc.2016.11.088

16. Hong SJ, Dawson TM, Dawson VL. Nuclear and mitochondrial conversations in cell death: PARP-1 and AIF signaling. Trends Pharmacol Sci. 2004;25(5):259-264. doi:10.1016/j.tips.2004.03.005

17. Um JH, Yun J. Emerging role of mitophagy in human diseases and physiology. BMB Rep. 2017;50(6):299-307. doi:10.5483/ BMBRep.2017.50.6.056

18. Zhang H. Targeting autophagy in lymphomas: a double-edged sword? Int J Hematol. 2018;107(5):502-512. doi:10.1007/s12185-018-2414-6

19. Kulikov AV, Luchkina EA, Gogvadze V, Zhivotovsky B. Mitophagy: link to cancer development and therapy. Biochem Biophys Res Commun. 2017;482(3):432-439. doi:10.1016/j.bbrc.2016.10.088

20. Chen Y, Chen HN, Wang K, et al. Ketoconazole exacerbates mitophagy to induce apoptosis by downregulating cyclooxygenase-2 in hepatocellular carcinoma. J Hepatol. 2019;70(1):66-77. doi:10.1016/ j.jhep.2018.09.022
21. Wang D, Qin J, et al. Potential allelopathic azaphilones produced by the endophytic Chaetomium globosum TY1 inhabited in Ginkgo biloba using the one strain-many compounds method. Nat Prod Res. 2017;31(6):724-728. doi:10.1080/14786419.2016.1217208

22. Oakes SA, Papa FR. The role of endoplasmic reticulum stress in human pathology. Annu Rev Pathol. 2015;10:173-194. doi:10.1146/ annurev-pathol-012513-104649

23. Green DR, Kroemer G. The pathophysiology of mitochondrial cell death. Science. 2004;305(5684):626-629. doi:10.1126/science.1099320

24. Gottlieb E, Armour SM, Harris MH, Thompson CB. Mitochondrial membrane potential regulates matrix configuration and cytochrome c release during apoptosis. Cell Death Differ. 2003;10(6):709-717. doi:10.1038/sj.cdd.4401231

25. Burke PJ. Mitochondria, bioenergetics and apoptosis in cancer. Trends Cancer. 2017;3(12):857-870. doi:10.1016/j.trecan.2017.10.006

26. Dombi E, Mortiboys H, Poulton J. Modulating mitophagy in mitochondrial disease. Curr Med Chem. 2018;25(40):5597-5612.

27. Kachalaki S, Ebrahimi M, Mohamed Khosroshahi L, Mohammadinejad S, Baradaran B. Cancer chemoresistance; biochemical and molecular aspects: a brief overview. Eur J Pharm Sci. 2016;89:20-30. doi:10.1016/j.ejps.2016.03.025

28. Turrini E, Ferruzzi L, Fimognari C. Natural compounds to overcome cancer chemoresistance: toxicological and clinical issues. Expert Opin Drug Metab Toxicol. 2014;10(12):1677-1690. doi:10.1517/ 17425255.2014 .972933

29. Crona DJ, Faso A, Nishijima TF, McGraw KA, Galsky MD, Milowsky MI. A systematic review of strategies to prevent cisplatin-induced nephrotoxicity. Oncologist. 2017;22(5):609-619. doi:10.1634/theoncologist.2016-0319

30. Lahera V, de Las Heras N, Lopez-Farre A, Manucha W, Ferder L. Role of mitochondrial dysfunction in hypertension and obesity. Curr Hypertens Rep. 2017;19(2):11. doi:10.1007/s11906-017-0710-9

31. Kafkova A, Trnka J. Mitochondria-targeted compounds in the treatment of cancer. Neoplasma. 2020;67(3):450-460. doi:10.4149/ neo_2020_190725N671

32. Shintani T, Klionsky DJ. Autophagy in health and disease: a double-edged sword. Science. 2004;306(5698):990-995. doi:10.11 26/science. 1099993

33. Tanaka A. Parkin-mediated selective mitochondrial autophagy, mitophagy: parkin purges damaged organelles from the vital mitochondrial network. FEBS Lett. 2010;584(7):1386-1392. doi:10.1016/j. febslet.2010.02.060
OncoTargets and Therapy

\section{Publish your work in this journal}

OncoTargets and Therapy is an international, peer-reviewed, open access journal focusing on the pathological basis of all cancers, potential targets for therapy and treatment protocols employed to improve the management of cancer patients. The journal also focuses on the impact of management programs and new therapeutic

\section{Dovepress}

agents and protocols on patient perspectives such as quality of life, adherence and satisfaction. The manuscript management system is completely online and includes a very quick and fair peer-review system, which is all easy to use. Visit http://www.dovepress.com/ testimonials.php to read real quotes from published authors. 